\title{
Aerosol delivery systems for treating obstructive airway diseases during the SARS-CoV-2 pandemic
}

\author{
Federico Lavorini ${ }^{1}\left[0\right.$. Omar S. Usmani ${ }^{2} \cdot$ Rajiv Dhand $^{3}$
}

Received: 30 January 2021 / Accepted: 20 July 2021 / Published online: 30 July 2021

(c) Società Italiana di Medicina Interna (SIMI) 2021

\begin{abstract}
The severe acute respiratory syndrome coronavirus 2 (SARS-CoV-2), which causes CoronaVirus Disease 2019 (COVID19), has resulted in a worldwide pandemic and currently represents a major public health crisis. It has caused outbreaks of illness through person-to-person transmission of the virus mainly via close contacts, and droplets produced by an infected person's cough or sneeze. Aerosolised inhaled therapy is the mainstay for treating obstructive airway diseases at home and in healthcare settings, but there is heightened particular concern about the potential risk for transmission of SARS-CoV-2 in the form of aerosolised respiratory droplets during the nebulised treatment of patients with COVID-19. As a consequence of this concern, the use of hand-held inhalers, especially pressurised metered dose inhalers, has risen considerably as an alternative to nebulisers, and this switch has led to inadequate supplies of inhalers in some countries. However, there is no evidence supporting an increased risk of viral transmission during nebulisation in COVID-19 patients. Furthermore, some patients may be unable to adequately use their new device and may not benefit fully from the switch to treatment via hand-held inhalers. Thus, there is no compelling reason to alter aerosol delivery devices for patients with established nebuliser-based regimens. The purpose of this paper is to discuss the current evidence and understanding of the use of aerosolised inhaled therapies during the SARS-CoV-2 pandemic and to provide some guidance on the measures to be taken to minimise the risk of transmitting infection, if any, during aerosol therapies.
\end{abstract}

Keywords Aerosol $\cdot$ Inhalers $\cdot$ Nebuliser $\cdot$ SARS-CoV-2 $\cdot$ Droplets $\cdot$ COVID-19

The Coronavirus disease 2019 (COVID-19) is a worldwide pandemic caused by the highly contagious novel severe acute respiratory syndrome coronavirus 2 (SARS-CoV-2) [1]. The latter belongs to the same class of coronaviruses that previously resulted in SARS and the Middle East Respiratory Syndrome, both of which infected many health care professionals in the course of providing patient care [1]. The SARS-CoV-2 virus is primarily transmitted through contact routes and dispersion of bio-aerosols from the patient [2]. Bio-aerosols are defined as aerosols or particulate and matter of animal, plant or microbial origin with a range from 0.1

Federico Lavorini

federico.lavorini@unifi.it

1 Department of Experimental and Clinical Medicine, University of Florence, Florence, Italy

2 Imperial College London and Royal Brompton Hospital, National Heart and Lung Institute, London, UK

3 Department of Medicine, University of Tennessee Graduate School of Medicine, Knoxville, TN, USA to $>100 \mu \mathrm{m}$ in diameter [3], that can contain viruses, bacteria, fungi, and are generated by infected persons when they cough, sneeze, talk, sing, or breathe [2]. They can land in the mouth, nose, or eyes of those in proximity, and they have the potential to be inhaled into the lungs. However, larger droplets in the cloud produced by coughing and sneezing settle quickly on surfaces around the infected subject, where they could be infectious by contact for several days [2]. The respiratory droplets evaporate to form smaller droplet nuclei that carry infectious agents, remain suspended in air, and transported over longer distance by airflow are highly respirable [2].

National and international guidelines recommend bioaerosol and contact precautions for those caring for COVID19 patients in ambulatory and acute care settings [1, 4-6], particularly during aerosol generating procedures, such as lung function and challenge testing, bronchoscopy, nebulised treatment, non-invasive ventilation, endotracheal intubation, placing patients into prone position, disconnecting the patient from the ventilator, tracheotomy, and 
cardiopulmonary resuscitation [7]. These procedures can result in the release of bio-aerosols from the respiratory tract when treating someone who is suspected or known to be suffering from an infectious agent transmitted wholly or partly by the airborne or droplet route [7]. Despite the lack of evidence, there is currently a heightened concern regarding the potential risk of transmission of SARS-CoV-2 in the form of aerosolised respiratory droplets from patients with COVID-19 undergoing nebulised treatment [8]. Furthermore, while some guidelines $[9,10]$ have advised against the use of nebuliser treatment unless absolutely necessary, others $[5,11]$ have recommended the continued use of nebulised treatment when applicable.

This intense debate with diametrically opposed opinions may have induced confusion and mis-interpretation among doctors thus encouraging them to avoid aerosol therapy in patients with COVID-19 and obstructive airway diseases treated at home, or to switch patients from nebulised therapies to portable inhalers. It seems worth recalling that a sudden switch to hand-held inhalers, particularly to pressurised metered-dose inhalers (pMDIs), may result in unintended consequences for some patients who may be unable to adequately use their new devices or benefit fully from treatment delivered by portable inhalers [12]

The present paper aims at providing guidance to physicians on aerosol therapies for the treatment of obstructive airway diseases in hospital or home settings during the SARS-CoV-2 pandemic, and, based on all current available information, illustrating measures to be taken to minimise the hypothetical risk of infection.

\section{Aerosol delivery systems and the risk of infection transmission}

Pressurised metered-dose inhalers, dry powder inhalers (DPIs), soft-mist inhalers (SMIs), and nebulisers are aerosol delivery systems used for administration of inhaled therapies to treat obstructive airway diseases such as asthma or chronic obstructive pulmonary disease [13]. Each of these delivery systems has advantages and limitations [13], as well as risks for device contamination [14-16] (Table 1). Although there is no ranking system that assesses the risk of transmission with various aerosol delivery devices, the rationale behind such an evaluation system could be the treatment time, the amount of emitted dose and aerosol mass generated by the device during aerosol therapy, and also the design of the device impacting towards the risk of contamination during device preparation, cleaning and its maintenance [2]. By design, the drug is enclosed in portable inhalers (i.e. pMDIs, DPIs, and SMIs); thus, they are hard to contaminate and the risk for viral transmission may be lower than nebulisers which are characterised by open medication reservoirs [15]. However, without cleaning the mouthpiece of portable inhalers, deposits could on the inner side of the mouthpiece, thus facilitating the growth of micro-organisms [16]. Bacterial

Table 1 Risk of device contamination and suggestions for safe delivery of aerosolised medications during COVID-19 pandemic

\begin{tabular}{|c|c|c|}
\hline Device & $\begin{array}{l}\text { Risk of con- } \\
\text { tamination }\end{array}$ & Suggestions for hand-held inhalers \\
\hline $\begin{array}{l}\text { pMDI } \\
\text { pMDI with } \\
\text { spacer or } \\
\text { VHC } \\
\text { DPI } \\
\text { SMI }\end{array}$ & $\begin{array}{l}\text { Low } \\
\text { Low }\end{array}$ & \multirow[t]{2}{*}{$\begin{array}{l}\text { Prefer the use pMDI in conjunction with a spacer or a VHC instead of the pMDI only } \\
\text { Use a spacer or a VHC with a mouthpiece instead of a face mask; } \\
\text { Train the patient to exhale into the spacer or the VHC to minimise exhaled aerosol dispersion to the environment; } \\
\text { Clean and disinfect the spacer or the VHC according to the manufacturer's guidelines; } \\
\text { Do not share pMDIs, spacers or VHCs with multiple patients; } \\
\text { Clean and disinfect pMDI canister and DPI/SMI mouthpiece with a } 70 \% \text { alcohol pad } \\
\text { Suggestions for nebulisers }\end{array}$} \\
\hline & & \\
\hline Nebulisers & High & $\begin{array}{l}\text { Wash hands and put fresh gloves before filling the nebuliser reservoir and administering treatments; } \\
\text { Ensure device is clean; } \\
\text { Avoid nebulisation in the presence of other people; } \\
\text { Carry out nebulisation near open windows or areas with adequate ventilation; } \\
\text { Ensure proper protective equipment for bystanders or health care personnel } \\
\text { Close the door while nebulisation is being undertaken; } \\
\text { Prefer a mouthpiece instead of a facemask } \\
\text { Prefer vibrating mesh over jet nebulisers; } \\
\text { Attach a filter to the expiratory port of the nebuliser } \\
\text { Rinse the nebuliser cup with distilled or sterile water } \\
\text { Keep the reservoir cap of mesh nebuliser closed after use; } \\
\text { Clean and disinfect the nebuliser according to the manufacturer's guidelines } \\
\text { Clean the surface and areas of nebulisation }\end{array}$ \\
\hline
\end{tabular}

pMDI pressurised metered-dose inhaler, $V H C$ valved-holding chamber, DPI dry powder inhaler, SMI soft mist inhaler 
contamination is common in uncleaned spacer devices used in the home setting [14]. Furthermore, the forceful inspirations required to aerosolise the medication from DPIs may cause cough [15] thus increasing the distance of exhaled air dispersion and the risk of viral transmission in the era of COVID-19. Therefore, some authors [15] recommend caution with the use of DPIs in patients with coughing and airway irritation. In summary, although there are no studies that have compared the risk of infection transmission between different portable inhalers or between portable inhalers and nebulisers, there appear to be a limited concern that portable inhalers increase SARS-CoV-2 transmission provided that the patient can use the device correctly.

Nebulisers are frequently used in hospital setting for treating acute asthma or COPD exacebations and at home in elderly patients unable to handle portable inhalers correctly [13]. It is far more appropriate to refer to the "nebuliser system" in its entirety [13] in which several components, other than the device itself, play a significant role in influencing aerosol delivery and the potential for microbial transmission [14]. Among the most influential are the compressor or line feed applied to the nebuliser, the volume fill, the residual volume, the driving gas flow, and the use of either a facemask or mouthpiece. Each of these significantly affects the total drug amount received by the patients during therapy, the rate of nebulized aerosol output, and the particle size distribution. If any component of the nebuliser system is replaced by another, then the nebuliser system has changed and the aerosol output characteristics will have been significantly altered [13]. At present, the evidence about the risk of viral transmission through aerosols generated by nebulisers is limited [17]. Aerosols generated by a nebuliser are derived from a medication solution that is not a bio-aerosol as that generated during coughing or sneezing [2]. However, nebulisers generate fugitive emissions from the device during the exhalation phase of the patient's breath $[15,18]$. The particle size of these fugitive emissions varies from 0.86 to $1.43 \mu \mathrm{m}$ and $\sim 50 \%$ of the generated aerosol is fugitive aerosol that remains suspended in the indoor environment for several hours [18]. Theoretically, a nebuliser could be considered as a fomite, and the risk of viral transmission with nebuliser therapies could be largely attributable to risk of contamination of the device itself. To investigate the hypothesis of virus spread during nebulisation, Tang and co-workers [19] simulated a spontaneously breathing adult patient with a heated manikin receiving nebulised therapy with a jet nebulizer and a facemask. Using a live-attenuated influenza vaccine as a surrogate virus tracer, the authors found that air samples obtained from three separate locations indicated 612 viruses per litre near the head, 174 viruses per litre near the abdomen, and 118 viruses per litre near the feet. These findings aerosols spreading at decreasing concentration with increasing distance from the head of the patient [19]. However, it is worth noting that these findings were obtained under strict laboratory conditions (fixed tidal volume, respiratory rate and exhalation flow, use of distilled water) that may not reflect what happen in a real-life setting. A retrospective pooled analysis on the risk of SARS-CoV transmission to health care professionals with various aerosol generating procedures showed that the risk of infection to health care professionals increased by 6.6 times with intubation, as opposed to 0.9 times with the use of nebulisers [7]. Unlike other aerosol generating procedures that carry contaminated particles derived from patients, the medication in the nebuliser is considered a non-patient source that might not generate bio-aerosols carrying pathogens, unless the nebuliser is contaminated [17]. Direct comparisons between different nebuliser types on the potential risk of microbial transmission are lacking, although some may be at greater risk than other. For example, jet nebulisers that are open to and positioned below the gas pathway could be contaminated by patient's secretions when they are directly connected to the patient interface through a mouthpiece or an endotracheal tube [17]. In contrast, by design vibrating mesh nebulisers generate aerosols via mesh plates that separate the medication from the patient interface [2]. In addition, mesh nebulisers have less residual volume $(<0.5 \mathrm{~mL})$ at the end of nebulisation than jet nebulisers (about $2 \mathrm{~mL}$ ) [13], which may create a less hospitable environment for micro-organisms. Therefore, vibrating mesh nebulisers could be preferred over jet nebulisers for aerosol therapies, although they need careful hygiene techniques as those of jet nebulisers.

Independent of the nebuliser type used, a risk of bioaerosol dispersion exists in case of contamination of the reservoir during medication loading process, which need to be performed using aseptic techniques. In suitable patients, a mouthpiece should be preferred over a facemask to improve treatment efficiency and reduce fugitive emissions because a mouthpiece does not force aerosols out of the interface during therapy [17]. Furthermore, placing a HEPA filter air cleaning system in the nebuliser's outlet in combination with a demistifier tent has been found to reduce fugitive emissions and exposure of health care professionals to aerosol medications [20]. Similarly, placing a filter in the expiratory limb of a ventilator circuit reduced escape of aerosols [11], thereby reducing the risk of transmission infection. Another investigation showed that, during adult breath simulations by means of a piston pum, a commercially available filter attached to a nebulizer reduced exhaled aerosol droplets between 0.06 and $0.1 \mu \mathrm{m}$ in size by $98 \%$ [21]. In summary, jet or mesh nebulisers should be used with a mouthpiece and a filter attached to the exhalation port of the nebuliser to reduce emission of exhaled aerosol droplets (Fig. 1). Care should be taken to ensure that the reservoir cap of the mesh nebuliser is closed after each use and the exhalation valve 


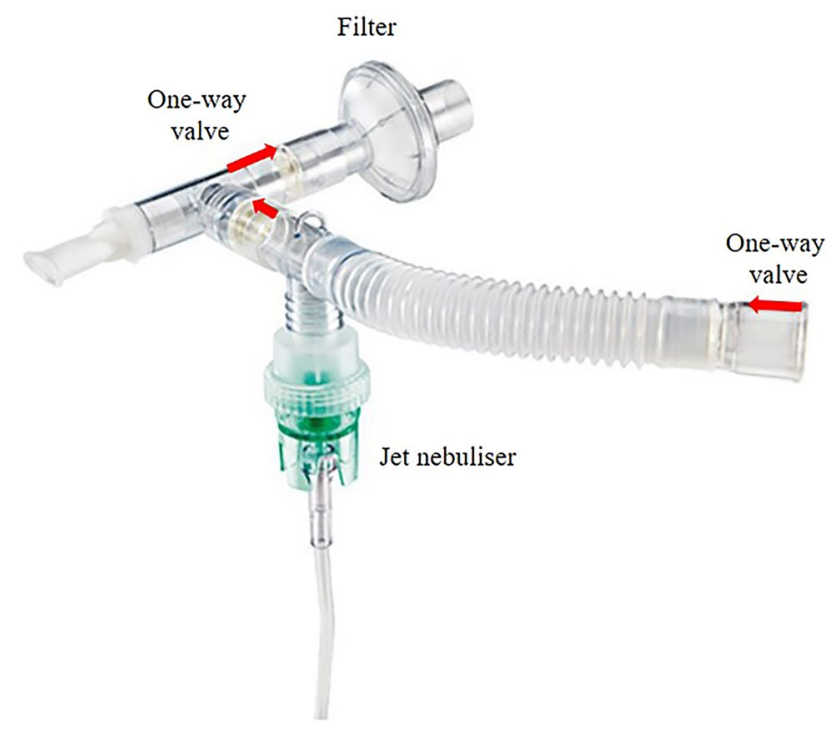

Fig. 1 Example of a filtered nebuliser set-up with one-way valves

of the mouthpiece is not blocked with the attachment of the filter to the mouthpiece. To avoid this problem, a silicon adaptor can be used between the filter and the mouthpiece of the mesh nebuliser.

\section{Strategies to promote a safe and effective use of portable inhalers and nebulisers during SARS-CoV-2 pandemic}

There is insufficient evidence on the risks associated with the transmission of SARS-CoV-2 during aerosol therapy. However, undertaking extra precautions to improve the safety and effectiveness of the aerosol delivery systems are essential during this global pandemic (Table 1). Ensuring device safety and effectiveness depends on selecting the most appropriate device for each patient after careful evaluation of device features, the potential risk for device contamination and viral transmission. The pMDI should always be used in conjunction with a dedicated spacer or a valved-holding chamber (VHC) and not transferred from patient to patient. A mouthpiece should be preferred over a facemask when using spacers/VHCs; the latter should be cleaned and disinfected according to the manufacturer's guidelines. Although not recommended, there are institutions that employ the common canister protocol, which is a cost-saving strategy used in hospitals and clinics to deliver pMDI medications or to teach pMDI technique using a placebo pMDI [22]. In both scenarios, the pMDI is reused between patients, along with an individual spacer/ VHC. In these cases, hand hygiene and wiping the pMDI with $70 \%$ alcohol before and after use between patients are recommended [14]. Similarly, wiping the DPI mouthpiece off with a dry clean cloth, such as a paper towel, and proper storage are necessary [14]. Nebulisers should be disposed of, rinsed air dried washed and sterilised between treatments using liquid/hospital-grade disinfectants. Nebuliser accessories can be cleaned and disinfected following manufacturer's instructions or using agents such as isopropanol 70 or hydrogen peroxide 3\% [14]. The use of one-way valves and filters, particularly high-efficiency air filters, is recommended with the nebulisers to minimise fugitive emissions and exposure of health care professionals (in hospital) and family members (at home) to aerosol medications. Finally, mouthpieces should be preferred while facemasks avoided as the delivery interface.

\section{Conclusions}

At present, evidence that nebulised treatment represents a risk for infection transmission is inconclusive due to the scarcity of information. Furthermore, there is no precedent to guide the treatment of patients with respiratory disease in the current situation. Although there is no evidence showing that aerosols generated by nebulisers contain pathogens unless the nebuliser is contaminated, clinicians should exercise caution with nebulisers and protect themselves from SARS-CoV-2 through a stringent sanitization protocol and the use of personal protective equipment during aerosol delivery to patients with known or suspected SARS-CoV-2 infection. Therefore, it is vital to train patients and clinicians on the risk of contamination during device preparation and viral transmission while providing suggestions on how to use each device safely and effectively in patients with SARS-CoV-2 infection. If a patient takes precautions, such as nebuliser hygiene, avoidance of nebuliser use in the presence of other people, ensure that nebuliser use is done near open window or in areas with adequate ventilation, the possible risks for bystanders and health care personnel could be minimised.

Author contributions All authors analysed the pertinent literature and had a say in the manuscript preparation, approval of the final version and the decision to submit for publication.

Funding This work was unfunded.

\section{Declarations}

Conflict of interest Federico Lavorini reports personal fees from AstraZeneca, Boehringer Ingelheim, Chiesi Farmaceutici, GlaxoSmithKline, HIKMA, Menarini International, Novartis, Orion, TEVA, Trudell International, research grants from AstraZeneca and GlaxoSmithKline, all of which are outside the submitted work. Omar S. Usmani reports grants and personal fees from AstraZeneca, Boehringer In- 
gelheim, Chiesi and GlaxoSmithKline, personal fees from Aerocrine, Cipla, Covis, DEVA, Napp, Menarini, Mundipharma, Orion, Sandoz, Takeda, Trudell International, Zentiva, and grants from Edmond Pharma, all of which are outside the submitted work. Rajiv Dhand reports personal fees from Astra-Zeneca, Boehringer-Ingelheim, Mylan, UptoDate, Teva, and research grant form Mylan, all of which are outside the submitted work.

Human and animal rights This manuscript is a point of view article and does not involve a research protocol requiring approval by the relevant institutional review board or ethics committee.

Informed consent For this type of study, formal consent is not required.

\section{References}

1. WHO Coronavirus disease (COVID-19) technical guidance: Infection prevention and control. Geneva: World Health Organization. 2020. https://www.who.int/emergencies/diseases/novel-coron avirus-2019/technical-gui dance/infection-prevention-and-control. Accessed 16 Mar 2020

2. Dhand R, Li J (2020) Coughs and sneezes: their role in transmission of respiratory viral infections, including SARS-CoV-2. Am J Respir Crit Care Med 202:651-659. https://doi.org/10.1164/rccm. 202004-1263PP

3. Douwes J, Thorne P, Pearce N, Heederik D (2003) Bioaerosol health effects and exposure assessment: progress and prospects. Ann Occup Hyg 47:187-200

4. American Association for Respiratory Care, Nebulizer CleaningFinal State Survey Worksheets Released. Updated: April 20, 2017. https://www.aarc.org/nebulizer-cleaning

5. National Institute for Health and Care Excellence, COVID19 rapid guideline: severe asthma (NICE guideline [NG166]) 2020.https://www.nice.or g.uk/guidance/ng 166

6. British Thoracic Society, COVID-19: information for the respiratory community. Advice about the safety of nebuliser use. https:// www.brit-thoracic.org.uk/about-us/covid-19-information-for-therespiratory-community

7. Tran K, Cimon K, Severn M, Pessoa-Silva CL, Conly J (2012) Aerosol generating procedures and risk of transmission of acute respiratory infections to healthcare workers: a systematic review. PLoS ONE 7(4):e35797

8. Amirav I (2020) Aerosol treatments during COVID19 pandemic: a personal journey. J Aer Med Pulm Drug Deliv 33:1-2

9. Global Initiative for Asthma, GINA Report, Global Strategy for Asthma Management and Prevention, 2020. https://ginasthma.
org/wp-content/uploads/2020/04/GINA-2020-full-report_-final-_ wms.pdf

10. Australian National Asthma Council, Managing asthma during the COVID-19 (SARS-CoV-2) pandemic, Available at: COVID-19, https://www.asthmahandbook.org.au/clinical-issues/covid-19

11. Fink JB, Ehrmann S, Li J, Dailey P et al (2020) Reducing aerosolrelated risk of transmission in the era of COVID-19: an interim guidance endorsed by the international society of aerosols in medicine. J Aerosol Med Pulm Drug Deliv 33:1-5

12. Lavorini F, Ninane V, Haughney J, Bjermer L et al (2013) Switching from branded to generic inhaled medications: potential impact on asthma and COPD. Expert Opin Drug Deliv 10:1597-1602. https://doi.org/10.1517/17425247.2013.852182

13. Lavorini F, Buttini F, Usmani OS (2019) 100 years of drug delivery to the lungs. Handb Exp Pharmacol 260:143-159

14. O'Malley CA (2015) Device cleaning and infection control in aerosol therapy. Respir Care 60:917-930

15. Cazzola M, Ora J, Bianco A, Rogliani P, Matera MG (2021) Guidance on nebulization during the current COVID-19 pandemic. Respir Med 176:106236

16. Harkins KJ (1999) Bacterial contamination of inhalers. J Hosp Infect 43:321

17. Ari A (2020) Promoting safe and effective use of aerosol devices in COVI-19: risks and suggestions for viral transmission. Exp Opin Drug Deliv 17:1509-1513

18. Harding H, Broom B, Broom J (2020) Aerosol generating procedures and infective risk to healthcare workers: SARS-CoV-2 the limits of evidence. J Hosp Infect 105:717-725

19. Tang JW, Kalliomaki P, Varila TM et al (2020) Nebulisers as a potential source of airborne virus. J Infect 81(4):647-679

20. Wittgen BP, Kunst PW, Perkins WR, Lee JK, Postmus PE (2006) Assessing a system to capture stray aerosol during inhalation of nebulized liposomal cisplatin. J Aerosol Med 19:385-391

21. Schuschnig U, Ledermüller R, Gramann J. Efficacy of the PARI filter-valve set to prevent environmental contamination with aerosol during nebulizer therapy. Preprint July 2020. : https://www. researchgate.net/publication/342987954

22. Larson T, Gudavalli R, Prater D, Sutton S (2015) Critical analysis of common canister programs: a review of cross functional considerations and health system economics. Curr Med Res Opin $31: 853$

Publisher's Note Springer Nature remains neutral with regard to jurisdictional claims in published maps and institutional affiliations. 